\title{
Exact perimeter generating function for a model of punctured staircase polygons
}

\author{
Iwan Jensen $\dagger$ and Andrew Rechnitzer \\ $\dagger$ Department of Mathematics and Statistics, The University of Melbourne, Victoria \\ 3010, Australia \\ $\ddagger$ Department of Mathematics, University of British Columbia, Vancouver, BC V6T \\ 1Z2, Canada \\ E-mail: I.Jensen@ms . unimelb.edu.au, andrewr@math.ubc.ca
}

\begin{abstract}
We have derived the perimeter generating function of a model of punctured staircase polygons in which the internal staircase polygon is rotated by a $90^{\circ}$ angle with respect to the outer staircase polygon. In one approach we calculated a long series expansion for the problem and found that all the terms in the generating function can be reproduced from a linear Fuchsian differential equation of order 4 . We then solved this ODE and found a closed form expression for the generating function. This is a highly unusual and most fortuitous result since ODEs of such high order very rarely permit a closed form solution. In a second approach we proved the result for the generating function exactly using combinatorial arguments. This latter solution allows many generalisations including to models with other types of punctures and to a model with any fixed number of nested rotated staircase punctures.
\end{abstract}

Submitted to: J. Phys. A: Math. Gen.

PACS numbers: $05.50 .+\mathrm{q}, 05.70 . J \mathrm{~J}, 02.10 . \mathrm{Ox}$ 


\section{Introduction}

Exactly solved models are special, important and relatively rare in physics. In many cases real life phenomena are modelled by simplified solvable models, which despite the simplifications can give us great insight into the behaviour of the more complicated fully-fledged problem. A well-known long standing problem in statistical mechanics is to find the perimeter generating function for self-avoiding polygons on a regular twodimensional lattice. Several simplifications of this problem are solvable [1], but all the simpler models impose an effective directedness or other constraint that reduces the problem, in essence, to a one-dimensional problem. A very important and interesting insight gained from these simple models (staircase polygons in particular) is the conjecture for the limit distribution of area moments and scaling function for selfavoiding polygons [2, 3, 4].

Here we report on the discovery of the exact perimeter generating function for a model of punctured staircase polygons. This solution was first conjectured on the basis of series analysis (see Sections 3 and 4) and subsequently proved using combinatorial arguments (Section 5). The combinatorial construction admits many generalisations including to models with other types of punctures and to a model with any fixed number of nested rotated staircase punctures (Section 6).

A staircase polygon can be viewed as the intersection of two directed walks starting at the origin, moving only to the right or up and terminating once the walks join at a vertex. The perimeter length of a staircase polygon is even. Let us denote by $c_{n}$ the number of staircase polygons of perimeter $2 n$. It is well known that $c_{n+1}=C_{n}=\frac{1}{n+1}\left(\begin{array}{c}2 n \\ n\end{array}\right)$ are given by the Catalan numbers $C_{n}$ and that the associated half-perimeter generating function is

$$
P(x)=\sum_{n} c_{n} x^{n}=\frac{1}{2}(1-2 x-\sqrt{1-4 x}) \propto(1-\mu x)^{2-\alpha},
$$

where the connective constant $\mu=4$ and the critical exponent $\alpha=3 / 2$. From this it readily follows that $c_{n}$ grows asymptotically as $c_{n} \sim A_{\mathrm{S}} \mu^{n} n^{\alpha-3}$, where the critical amplitude $A_{\mathrm{S}}=-1 /(2 \Gamma(\alpha-2))=1 /(4 \sqrt{\pi}) \approx 0.141$.

Punctured staircase polygons [5] are staircase polygons with internal holes which are also staircase polygons (the polygons and holes are mutually- as well as self-avoiding). In a recent paper [6] we studied the problem of staircase polygons with a single hole and found that the perimeter generating function can be expressed as the solution of an 8th order linear ODE. Here we will study the case with a single hole but where the internal polygon is rotated by $90^{\circ}$ with respect to the main axis. So the internal staircase polygon is the intersection of two walks starting at a vertex (the top left-most vertex of the internal polygon) and taking steps only to the right and down. We will refer to these objects as rotated punctured staircase polygons. In Figure 1 we have shown an example of each of the two types of punctured polygons. The perimeter length of a punctured polygon is the sum of the outer perimeter and the perimeter of the hole. We denote by $p_{n}$ the number of punctured staircase polygons of perimeter $2 n$ and by 

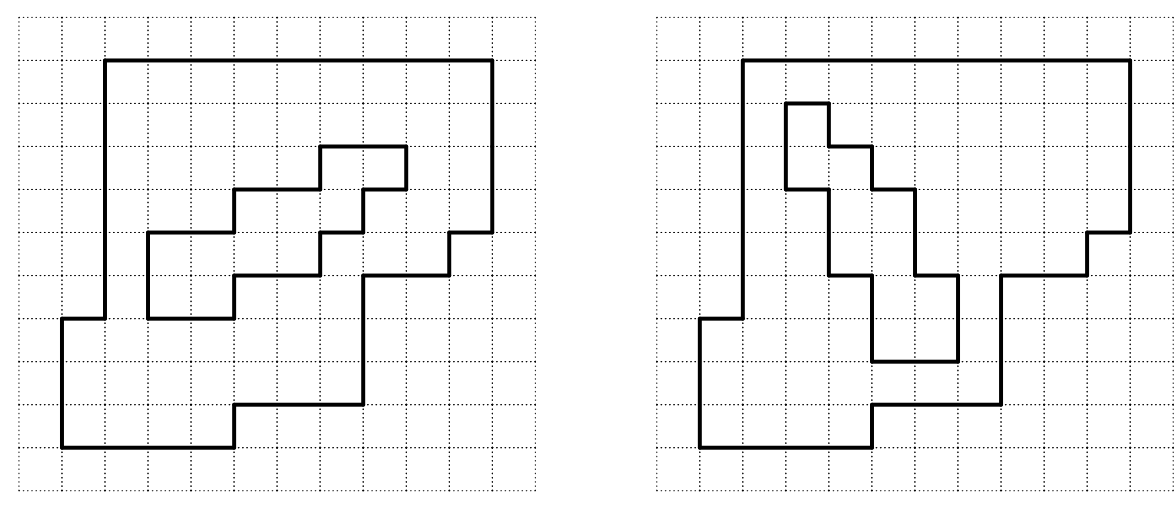

Figure 1. The left panel shows a typical punctured staircase polygon and the right panel a rotated punctured staircase polygon.

$r_{n}$ the number of rotated punctured staircase polygons of perimeter $2 n$. The associated generating functions are $\mathcal{P}(x)=\sum p_{n} x^{n}$ and $\mathcal{P}_{\text {Rot }}(x)=\sum r_{n} x^{n}$, respectively.

It is intuitively clear that since staircase polygons are elongated along the diagonal of growth there are many more restrictions on the placement of the rotated polygon and hence we expect $r_{n} \leq p_{n}$. The difference between the two cases can be made more explicit by noting that any polygon has a minimal bounding rectangle which is the smallest rectangle completely containing the polygon. In the rotated case the outer polygon is excluded from the minimal bounding rectangle of the hole while in the aligned case the outer polygon may enter the minimal bounding rectangle of the hole (as is the case in the left panel of Figure 1).

In [5] it was proved that the connective constant $\mu$ of $k$-punctured polygons (polygons with $k$ holes) is the same as the connective constant of unpunctured polygons. Numerical evidence clearly indicated that the critical exponent $\alpha$ increased by $3 / 2$ per puncture (this was proved for a single puncture and conjectured in the general case). In recent work Richard, Jensen and Guttmann [7, Theorem 2] proved the exponent formula for a finite number of punctures and proved an exact formula for the leading amplitude of punctured staircase polygons. It it worth noting that the proofs in these papers never considered restrictions on the placement of the internal polygon, that is, the hole could be placed in any way one pleases. The results, therefore, carry over unaltered to the problem of rotated punctured staircase polygons. Interestingly, this means that the leading asymptotic forms of $r_{n}$ and $p_{n}$ are exactly the same; any differences arise only from sub-dominant correction terms. In particular the dominant singular behaviour of $\mathcal{P}(x)$ and $\mathcal{P}_{\text {Rot }}(x)$ is a simple pole at $x=x_{c}=1 / 4$ and thus $p_{n} \sim A_{\mathrm{P}} 4^{n}$ and $r_{n} \sim A_{\mathrm{R}} 4^{n}$, where we expect that $A_{\mathrm{P}}=A_{\mathrm{R}}$.

The rest of the paper is organised as follows. In Section 2 we briefly describe the algorithm used to count the number of punctured polygons. Section 3 describes how we found the underlying ODE and gives a brief review of its critical properties. Then, in Section 4, we give the closed form solution for the generating function. Section 5 contains 
Table 1. The number $r_{n}$ of rotated punctured staircase polygons.

\begin{tabular}{llllll}
\hline$n$ & $r_{n}$ & $n$ & $r_{n}$ & $n$ & $r_{n}$ \\
\hline 8 & 1 & 19 & 201099320 & 30 & 1761048979430768 \\
9 & 12 & 20 & 889594210 & 31 & 7344148372848448 \\
10 & 94 & 21 & 3896177956 & 32 & 30553399525543917 \\
11 & 604 & 22 & 16920602244 & 33 & 126830292729207600 \\
12 & 3461 & 23 & 72954802376 & 34 & 525432597401411262 \\
13 & 18412 & 24 & 312595497011 & 35 & 2172784129140676636 \\
14 & 93016 & 25 & 1332153819572 & 36 & 8969907982862433143 \\
15 & 452500 & 26 & 5650155211024 & 37 & 36973458557889104804 \\
16 & 2139230 & 27 & 23864065957572 & 38 & 152186202561672129880 \\
17 & 9890404 & 28 & 100418115489408 & 39 & 625590993787945461804 \\
18 & 44921002 & 29 & 421151065542880 & 40 & 2568489385305061560252 \\
\hline
\end{tabular}

an outline of the rigorous combinatorial derivation of the generating function while Section 6 contains a brief outline of results obtained by generalising the combinatorial formula. Finally, in Section 7 we briefly discuss our findings.

\section{Computer enumeration}

Rotated punctured staircase polygons were counted using a generic algorithm designed to count punctured convex polygons, which in turn was based on an algorithm for counting general self-avoiding polygons [8], but with restrictions so as to limit the count to various types of convex polygons. A polygon is convex if the perimeter is equal to that of its minimal bounding rectangle (the smallest rectangle into which one can fit the polygon). A staircase polygon is a restricted convex polygon and is produced by demanding that two diagonally opposite corners of the minimal bounding rectangle are part of the convex polygon. A standard punctured staircase polygon is then produced by demanding that the outer and inner polygons must include, say, the lower left and upper right corners of their respective minimal bounding rectangles. For rotated punctured staircase polygons the inner polygon must include the upper left and lower right corners as illustrated in Figure 1. The algorithm used to count punctured staircase polygons (the aligned case) is described in [6, 9].

Using this algorithm we calculated $r_{n}$ up to half-perimeter $n=125$. Since the smallest punctured polygon has half-perimeter 8 this gives us a 118 non-zero terms. In Table 1 we have listed the first few values of $r_{n}$. For comparison we mention that the first discrepancy with $p_{n}$ is at $n=12$ and $p_{12}-r_{12}=2$. Also note, for comparison, that $p_{40} / r_{40} \approx 1.041$. 


\section{The Fuchsian differential equation}

Recently Zenine et al [10, 11, 12] used series analysis to obtain linear ODEs satisfied by the 3 - and 4-particle contributions $\chi^{(3)}$ and $\chi^{(4)}$ to the Ising model susceptibility. In [6, 9] we used their method to find linear ODEs for the perimeter generating functions of punctured staircase polygons and three-choice polygons. We have used this technique to find a linear ODE satisfied by the generating function $\mathcal{P}_{\text {Rot }}(x)$ for rotated punctured staircase polygons. We briefly outline the method here. Starting from a (long) series expansion for a function $G(x)$ we look for a linear differential equation of order $m$ of the form

$$
\sum_{k=0}^{m} P_{k}(x) \frac{\mathrm{d}^{k}}{\mathrm{~d} x^{k}} G(x)=0,
$$

such that $G(x)$ is a solution to this homogeneous linear differential equation, where the $P_{k}(x)$ are polynomials. In order to make it as simple as possible we start by searching for a Fuchsian [13] equation. Such equations have only regular singular points. Computationally the Fuchsian assumption simplifies the search for a solution. From the general theory of Fuchsian [13] equations it follows that the degree of $P_{k}(x)$ is at most $n-m+k$ where $n$ is the degree of $P_{m}(x)$. To simplify matters further (reduce the order of the unknown polynomials) it is advantageous to explicitly assume that the origin and $x=x_{c}=1 / 4$ are regular singular points and to set $P_{k}(x)=Q_{k}(x) S(x)^{k}$, where $S(x)=x(1-4 x)$. Thus when searching for a solution of Fuchsian type there are only two parameters, namely the order $m$ of the differential equation and the degree $q_{m}$ of the polynomial $Q_{m}(x)$. Since the degree of the imposed factor $S(x)$ is 2 the restriction on the degree of $P_{k}(x)$ means that the degree of $Q_{k}(x)$ is at most $q_{m}+m-k$. The number of

unknown coefficients is thus $L=\sum_{j=0}^{m}\left(q_{m}+j+1\right)-1=(m+1) q_{m}+(m+2)(m+1) / 2-1$, where we get one less unknown by demanding that the leading coefficient in $Q_{m}(x)$ is 1 .

We then search systematically for solutions by varying $m$ and $q_{m}$. In this way we found a solution with $m=4$ and $q_{m}=4$, which required the determination of only $L=34$ unknown coefficients. We have 118 non-zero terms in the half-perimeter series and thus have 84 additional terms with which to check the correctness of our solution. This should be contrasted with punctured staircase polygons [6] where we first found a solution with $m=10$ and $q_{m}=11$, which required the determination of $L=186$ unknown coefficients. The lowest order ODE we found had order $m=8$ with $q_{m}=27$, which requires the determination of 287 unknown coefficients. So clearly the restrictions imposed by the rotation of the internal polygon results in a much simpler problem ( $a$ priori there was obviously no reason to believe this would be the case).

The (half)-perimeter generating function $\mathcal{P}_{\text {Rot }}(x)$ for rotated punctured staircase polygons is a solution to the linear differential equation of order 4

$$
\sum_{k=0}^{4} P_{n}(x) \frac{\mathrm{d}^{k}}{\mathrm{~d} x^{k}} \mathcal{P}_{\text {Rot }}(x)=0
$$


Table 2. Critical exponents for the regular singular points of the Fuchsian differential equation satisfied by $\mathcal{P}_{\text {Rot }}(x)$.

\begin{tabular}{lllll}
\hline Singularity & \multicolumn{3}{l}{ Exponents } \\
\hline$x=0$ & -8 & -7 & -4 & 0 \\
$x=1 / 4$ & -1 & $-3 / 4$ & $-1 / 2$ & $-1 / 4$ \\
$x=-3 / 4$ & 0 & $1 / 2$ & 1 & 2 \\
$1 / x=0$ & 6 & $3 / 2$ & 7 & $15 / 2$ \\
$Q_{4}(x)=0$ & 0 & 1 & 2 & 3 \\
\hline
\end{tabular}

with

$P_{4}(x)=x^{2}(1-4 x)^{4}(3+4 x)\left(-252+300 x+2365 x^{2}+1800 x^{3}\right)$,

$P_{3}(x)=x(1-4 x)^{3}\left(5292+1872 x-56127 x^{2}-115700 x^{3}-97280 x^{4}-28800 x^{5}\right)$,

$P_{2}(x)=24(1-4 x)^{2}\left(-378-189 x+5565 x^{2}+4085 x^{3}+1480 x^{4}+4090 x^{5}+3600 x^{6}\right)$,

$P_{1}(x)=24(1-4 x)\left(-126-1113 x+1250 x^{2}+24540 x^{3}-2805 x^{4}-44960 x^{5}-28800 x^{6}\right)$,

$P_{0}(x)=24\left(504+672 x-12200 x^{2}-38475 x^{3}+112600 x^{4}+228800 x^{5}+115200 x^{6}\right)$.

The singular points of the differential equation are given by the roots of $P_{4}(x)$. One can easily check that all the singularities (including $x=\infty$ ) are regular singular points so equation (3) is indeed of the Fuchsian type. It is thus possible using the method of Frobenius to obtain from the indicial equation the critical exponents at the singular points. These are listed in Table 2.

We shall now consider the local solutions to the differential equation around each singularity. Recall that in general it is known [14, 13] that if the indicial equation yields $k$ critical exponents which differ by an integer, then the local solutions may contain logarithmic terms up to $\log ^{k-1}$. However, we have found in the analysis of previous problems that for the Fuchsian equations of the type described by equation (3) only multiple roots of the indicial equation give rise to logarithmic terms in the local solution around a given singularity, so that a root of multiplicity $k$ gives rise to logarithmic terms up to $\log ^{k-1}$. In particular this means that near any of the 3 roots of $Q_{4}(x)=-252+300 x+2365 x^{2}+1800 x^{3}$, the local solutions have no logarithmic terms and the solutions are thus analytic since all the exponents are positive integers. The roots of $Q_{4}(x)$ are thus apparent singularities [13, 14] of the Fuchsian equation (3). This will become completely self-evident in the next section where we present a closed form solution of $\mathcal{P}_{\text {Rot }}(x)$. So the points of interest are the physical critical point $x=x_{c}=1 / 4$, where the dominant singularity is a simple pole, modified by 3 correction terms with exponents that increase in steps of $1 / 4$. At the non-physical critical point $x=-3 / 4$ the function has a simple square root singularity. 


\section{The solution to the ODE}

Given an ODE it is often useful to look for simple solutions. In this case we first looked for solutions of the form $P(x) /(1-4 x)^{\gamma}$, where $P(x)$ is a polynomial and $\gamma=1,3 / 4,1 / 2$ or $1 / 4$. In this fashion we discovered the solutions $F_{1}(x)$ and $F_{2}(x)$ listed below. This gave us great hope that we could find a solution to the full problem since the two simple solutions can be used to write the ODE as a product of three differential operators of order 2, 1 and 1, respectively. As it turned out the dsolve package in Maple was up to the task and readily found four solutions, including $F_{1}(x)$ and $F_{2}(x)$. The remaining two solutions as supplied by dsolve were quite complicated expressions, but with a bit of work we managed to simplify (largely by hand) to the expressions $F_{3}(x)$ and $F_{4}(x)$ given below.

The four linearly independent solutions to the ODE are:

$$
\begin{aligned}
& F_{1}(x)=\frac{1-8 x+16 x^{2}-4 x^{3}}{1-4 x} \\
& F_{2}(x)=\frac{1-6 x+6 x^{2}}{\sqrt{1-4 x}} \\
& F_{3}(x)=\frac{1}{\sqrt{2}} \frac{\sqrt{2+\sqrt{3+4 x}}\left(3-8 x+2 x^{2}-\sqrt{3+4 x}(1-2 x)\right)}{(1-4 x)^{3 / 4}} \\
& F_{4}(x)=\frac{1}{\sqrt{2}} \frac{\left(3-8 x+2 x^{2}+\sqrt{3+4 x}(1-2 x)\right)}{(1-4 x)^{1 / 4} \sqrt{2+\sqrt{3+4 x}}}
\end{aligned}
$$

and the generating function is simply

$$
\mathcal{P}_{\text {Rot }}(x)=-\frac{1}{4}\left[F_{1}(x)-F_{2}(x)+F_{3}(x)-F_{4}(x)\right] ;
$$

we prove this formula in the next section. Obviously, $\mathcal{P}_{\text {Rot }}(x)$ is dominated asymptoti-

cally by $-\frac{1}{4} F_{1}(x)$, so the leading amplitude $A_{\mathrm{R}}=-\frac{1}{4}\left[(1-4 x) F_{1}(x)\right]_{x=1 / 4} / \Gamma(1)=1 / 64$, which is exactly the same as the leading amplitude of punctured staircase polygons and equal to the proved formula [7].

\section{Combinatorial construction of a staircase polygon with a rotated staircase hole}

Before we consider the construction, it is important to note is that because the inner polygon (the hole) is rotated $90^{\circ}$ from the outer polygon, it follows that the outer polygon must avoid not only the inner polygon, but also its minimal bounding rectangle. This considerably simplifies the problem.

We build the punctured polygon in four steps (see Figure 2).

a: Construct the inner polygon, enumerating it by total perimeter, width and height. A wasp-waist decomposition or a column-by-column construction gives the following 


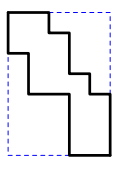

a

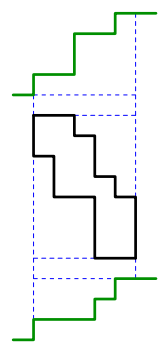

b

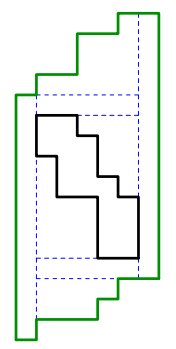

C

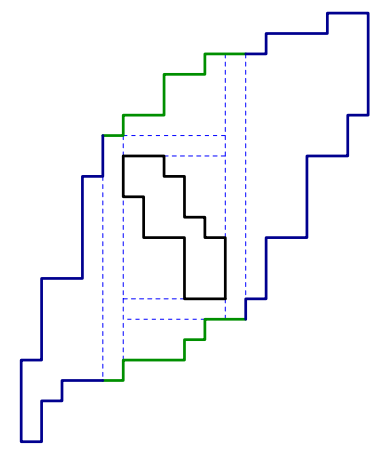

d

Figure 2. The four stages in the combinatorial construction of a rotated staircase polygon. We start (a) from a staircase polygon sitting in it's minimal bounding rectangle. Next (b) we attach directed walks above and below the rectangle and (c) connect the walks to form an outer polygon. Finally (d) we complete the construction by attaching staircase factors on the left and right sides of the outer polygon.

generating function [1]:

$$
\begin{aligned}
& P(w, h ; x, y)=x^{2} y^{2} w h+\left(x^{2} w+y^{2} h\right) P(w, h ; x, y)+P(w, h ; x, y)^{2} \\
& =\frac{1}{2}\left(1-x^{2} w-y^{2} h-\sqrt{1-2 x^{2} w-2 y^{2} h+x^{4} w^{2}-x^{2} y^{2} w h+y^{4} h^{2}}\right)
\end{aligned}
$$

where $x, y$ are conjugate to the number of horizontal and vertical bonds and $w, h$ are conjugate to the width and height of the associated minimum bounding rectangle.

b: We then attach two directed paths to the bounding rectangle of the polygon; one above and one below. If the polygon has width $w$, then each of these paths must start and end with a horizontal bond, contain a total of $w+2$ horizontal bonds. The generating function of a directed path is

$$
D(x, y)=\frac{1}{1-x-y}=\sum_{n} \frac{x^{n}}{(1-y)^{n+1}} .
$$

Hence the generating function of directed walks with exactly $w$ horizontal bonds is $x^{w} /(1-y)^{w+1}$. So if we wish to attach a directed path of width $w+2$ above a staircase polygon of width $w$, then the corresponding generating function is $P(w x /(1-y), h ; x, y) \cdot x^{2} /(1-y)$. If we attach two directed paths (one above and one below) we obtain $P\left(w x^{2} /(1-y)^{2}, h ; x, y\right) \cdot x^{4} /(1-y)^{2}$.

Next we have to insert a (positive) number of rows between the directed paths and the bounding rectangle. We also need to keep track of some extra variables. In particular we need to know the height of the leftmost and rightmost columns (the distances between the end-points of the directed walks). Let these be counted by the variables $s$ and $t$, respectively.

Inserting a positive number of rows between the directed paths and the bounding rectangle is equivalent to multiplying by $\left(\frac{s t}{1-s t}\right)^{2}$. Since each directed path adds to either the height of the leftmost or rightmost column we need to modify what we 
did above. In particular the generating function of the upper directed path becomes $(1-x-y t)^{-1}$ and that of the lower path becomes $(1-x-y s)^{-1}$. Thus we arrive at

$Q(s, t ; x, y)=\left(\frac{s t}{1-s t}\right)^{2} \frac{x^{4}}{(1-s y)(1-t y)} P\left(\frac{x^{2}}{(1-s y)(1-t y)}, s t, x, y\right)$

where $Q$ is the generating function of open configurations as drawn in Figure 2(b).

c: I the next step we simply add vertical bonds to each of the open ends thus connecting the directed paths so as to form a closed object. This corresponds to mapping $s \mapsto s y$ and $t \mapsto t y$. Hence our generating function is

$$
\begin{aligned}
& R(s, t ; x, y)=Q(s y, t y ; x, y) \\
& =\left(\frac{s t y^{2}}{1-s t y^{2}}\right)^{2} \frac{x^{4}}{\left(1-s y^{2}\right)\left(1-t y^{2}\right)} P\left(\frac{x^{2}}{\left(1-s y^{2}\right)\left(1-t y^{2}\right)}, s t y^{2}, x, y\right)
\end{aligned}
$$

which counts the closed configurations as drawn in Figure 2(c).

d: The punctured staircase polygons are completed by adding left and right staircase polygon factors to each side. Roughly speaking, this is done by substituting $s \mapsto \sigma(x, y)$ and $t \mapsto \sigma(x, y)$ where $\sigma$ is very nearly the staircase polygon generating function.

More specifically we do a Temperley/Bousquet-Mélou-like column-by-column construction to obtain the appropriate generating function. In [1] the functional equation used to enumerate staircase polygons by perimeter and area is given by

$$
F(s)=A(s)+\frac{x^{2} s}{(1-s)\left(1-s y^{2}\right)}(F(1)-F(s))
$$

where $A(s)$ is the "seed" configuration to which we append columns and $F(s)$ is the generating function of the resulting staircase polygons.

This functional equation can be solved using the kernel method. First take all the $F(s)$ terms to the left-hand side of the equation:

$$
F(s)\left(1+\frac{x^{2} s}{(1-s)\left(1-s y^{2}\right)}\right)=A(s)+\frac{x^{2} s}{(1-s)\left(1-s y^{2}\right)} F(1)
$$

Now set $s$ to a value that takes the coefficient of $F(s)$ (the kernel) to be zero. This value is

$$
\sigma(x, y)=\frac{1}{2 y^{2}}\left(1-x^{2}+y^{2}-\sqrt{1-2 x^{2}-2 y^{2}-2 x^{2} y^{2}+x^{4}+y^{4}}\right)
$$

which is very nearly the staircase polygon generating function. Setting $s$ to this value leaves

$$
0=A(\sigma)+\frac{x^{2} \sigma}{(1-\sigma)\left(1-\sigma y^{2}\right)} F(1)=A(\sigma)-F(1)
$$

where we have made use of the fact that $x^{2} \sigma=-(1-\sigma)\left(1-\sigma y^{2}\right)$. We do not care about $F(s)$ and only want $F(1)$ (i.e. we do not need to know the leftmost and rightmost column heights of our punctured polygons) and we are done. 
The generating function of staircase polygons with a rotated staircase hole is given by

$S(x, y)=R(\sigma, \sigma ; x, y)=\left(\frac{\sigma^{2} y^{2}}{1-\sigma^{2} y^{2}}\right)^{2} \frac{x^{4}}{\left(1-\sigma y^{2}\right)^{2}} P\left(\frac{x^{2}}{\left(1-\sigma y^{2}\right)^{2}}, \sigma^{2} y^{2}, x, y\right)$

Of course, the punctured polygons always have an even number of horizontal and vertical bonds, so we can replace $x \mapsto \sqrt{x}$ and $y \mapsto \sqrt{y}$ to obtain the half-perimeter generating function:

$$
S(x, y)=\left(\frac{\sigma^{2} y}{1-\sigma^{2} y}\right)^{2} \frac{x^{2}}{(1-\sigma y)^{2}} P\left(\frac{x}{(1-\sigma y)^{2}}, \sigma^{2} y, x, y\right)
$$

where $P$ is the solution of $P=x y w h+(x w+y h) P+P^{2}$ and $\sigma$ is the solution of $(1-\sigma)(1-y \sigma)+x \sigma=0$. One may verify that setting $y=x$ does indeed recover the generating function given in equation (6)

\section{Generalisations of the combinatorial results}

Here we shall consider three generalisations of the combinatorial result. In the first generalisation we look at the case where we do not count the perimeter of the hole (in other words the perimeter of the punctured polygon is equal to the perimeter of the external polygon only). In the second generalisation we look at what happens if we replace the internal polygon by a convex polygon of a different type. In the third generalisation we show that the formula (16) can be used to derive the generating function for nested staircase polygons.

The first generalisation is simply obtained by noting that in formula (16) the variables $x$ and $y$ 'counting' the perimeter can be separated in order to count the external and internal perimeter. In fact going back through the derivation we note that in equation (16) only the last (right-most) occurrence of $x$ and $y$ come from the internal polygon (all the other occurrences arise from the outer polygon). We thus find that the generating function for rotated punctured staircase polygons counted only by external perimeter is

$$
S(x, y)=\left(\frac{\sigma^{2} y}{1-\sigma^{2} y}\right)^{2} \frac{x^{2}}{(1-\sigma y)^{2}} P\left(\frac{x}{(1-\sigma y)^{2}}, \sigma^{2} y, 1,1\right)
$$

Analysis of this solution shows that again it is the solution to a 4th order ODE and there is a major change in the critical behaviour. The function now has a square root singularity at $x=4 / 25$, that is a behaviour $\propto \sqrt{4-25 x}$, so the connective constant changes from 4 to $25 / 4$. There is still a singularity at $x=1 / 4$, and interestingly the behaviour around it is quite complicated with exponents $-1,-1 / 2,0$ and $1 / 2$.

The second generalisation is obtained by noting that in the derivation of formula (16) we never really used the fact that the internal polygon was a staircase polygon we only relied on the fact that the external polygon is excluded from the minimal bounding rectangle of the hole. This means that we can replace $P(w, h, x, y)$ with the generating function of another type of polygon provided we know how to enumerate 
Table 3. The critical point and exponents of staircase polygons with a puncture which is a convex polygon. The left-most columns give the behaviour of the convex polygon used for the puncture while the right-most columns is the behaviour once 'wrapped' in an outer staircase polygon.

\begin{tabular}{lll|ll}
\hline Puncture type & $x_{c}$ & Exponent & $x_{c}$ & Exponent \\
\hline Rectangle & 1 & -2 & $1 / 4$ & $-1,-1 / 2$ \\
Ferrers & $1 / 2$ & -1 & $1 / 4$ & $-1,-1 / 2$ \\
Stack & $(3-\sqrt{5}) / 2$ & -1 & $1 / 4$ & $-1,-1 / 2$ \\
Staircase & $1 / 4$ & $1 / 2$ & $1 / 4$ & $-1,-3 / 4,-1 / 2,-1 / 4$ \\
Directed & $1 / 4$ & $-1 / 2$ & $1 / 4$ & $-5 / 4,-3 / 4$ \\
Convex & $1 / 4$ & $-2,-3 / 2$ & $1 / 4$ & $-2,-7 / 4,-3 / 2,-5 / 4$ \\
\hline
\end{tabular}

them by width, height and perimeter. In Table 3 we summarise the results for the critical behaviour at the leading singularity when the puncture is a convex polygon. Firstly we note that the dominant singularity remains at $x=1 / 4$. However, the critical exponent changes depending on the asymptotic growth of the generating function of the puncture. When the asymptotic growth of the puncture is slower than or equal to that of staircase polygons we have a simple pole at $x=1 / 4$. However, in the case of directed convex and the case of convex polygons (both of which are asymptotically more numerous than staircase polygons) the critical exponent changes to reflect a faster growth in the number of the associated punctured polygons.

The third generalisation is obtained by noting that formula (16) can be iterated. This gives us (at least in principle) solutions for any fixed number of staircase polygons nested within one another. Where at each level the outer polygon avoids the minimal bounding rectangle of the inner polygon(s). In order to prove the iteration procedure we note the following facts:

(i) As already noted above the $x$ and $y$ variables can easily be distinguished so as to count the outer and inner perimeters separately.

(ii) We don't really need to keep track of the internal perimeter in detail.

(iii) Because of the convexity constraint the $(x, y)$-variables from the outer polygon automatically counts the width and height so the $w$ and $h$ variables in the original $P$ are not really needed.

(iv) We have no restriction on what we put inside the hole. In particular we could put a staircase polygon with a rotated staircase polygon into the hole.

With this in mind we can see formula (16) as an operator on generating functions along the lines of "wrap me in a staircase polygons but don't enter my minimal bounding rectangle",

$$
T[P(x, y)] \mapsto B(x, y) P\left(x z /(1-\sigma y)^{2}, \sigma^{2} y z\right),
$$


where $B(x, y)$ is the prefactor $\left(\sigma^{2} y /\left(1-\sigma^{2} y\right)\right)^{2} x^{2} /(1-\sigma y)^{2}$. By iteration we then find that the generating function for $k$-nested rotated staircase polygons is

$$
\mathcal{P}_{k}(x, y)=T\left[\mathcal{P}_{k-1}(x, y)\right] \text {, with } \mathcal{P}_{0}(x, y)=P(x, y) .
$$

The solution for the twice nested case $\mathcal{P}_{2}(z)=\mathcal{P}_{2}(z, z)$ has been confirmed by enumeration results. The formula (19) gives us (at least in principle) solutions for any fixed number of staircase polygons nested within one another. In reality the solutions are pretty nasty and so far we have determined mainly the leading asymptotic behaviour. In summary we find that the generating functions $\mathcal{P}_{k}(z)=\mathcal{P}_{k}(z, z)$ have a singularity at $z=1 / 4$ with leading exponent $-2+1 / 2^{k-1}$. We have confirmed the exponent value for $k$ up to 10 by doing formal expansions around $z=1 / 4$. It appears that the singularity is of order $2^{k+1}$ and that the full set of exponents is given by $-2+1 / 2^{k-1}+j / 2^{k+1}$,

$j=0, \ldots, 2^{k+1}-1$. We have already seen that this behaviour is true for $\mathcal{P}_{1}(z)$ from the closed formed solution. For $k=2$ we took the formula for $\mathcal{P}_{2}(z)$ and expanded in a series to order 1000. Using the method described in Section 3 we managed to find the exact ODE which is of order 8 as expected with degree of the leading polynomial equal to 66 . Solving the indicial equation confirms that the exponents at $z=1 / 4$ are $-12 / 8$, $-11 / 8,-10 / 8,-9 / 8,-8 / 8,-7 / 8,-6 / 8$, and $-5 / 8$ in complete agreement with our conjecture.

\section{Summary and Discussion}

Using series expansions for rotated punctured staircase polygons we found that the half-perimeter generating function $\mathcal{P}_{\text {Rot }}(x)$ satisfies a fourth order Fuchsian ODE. We then solved this ODE and found a closed form solution for $\mathcal{P}_{\text {Rot }}(x)$. The solution is dominated by a function $-F_{1}(x) / 4$ with a simple root at $x=x_{c}=1 / 4$. There are three sub-dominant correction terms $-F_{3}(x) / 4$ which has critical exponent $-3 / 4, F_{2}(x) / 4$ with exponent $-1 / 2$ and $F_{4}(x) / 4$ with exponent $-1 / 4$. In addition there is a square root singularity at $x=-3 / 4$.

This should be compared to our analysis [6] of the ODE satisfied by the generating function $\mathcal{P}(x)$ for punctured staircase polygons, which showed that near the physical critical point $x=x_{c}=1 / 4$

$$
\mathcal{P}(x) \sim \frac{A(x)}{(1-4 x)}+\frac{B(x)+C(x) \log (1-4 x)}{\sqrt{1-4 x}},
$$

where $A(x), B(x)$ and $C(x)$ are analytic in a neighbourhood of $x_{c}$. In addition $\mathcal{P}(x)$ has a singularity on the negative $x$-axis, at $x=x_{-}=-1 / 4$ with the singular behaviour

$$
\mathcal{P}(x) \sim D(x)(1+4 x)^{13 / 2},
$$

where again $D(x)$ is analytic near $x_{-}$. Finally, the ODE also had a pair of singularities at $x= \pm \mathrm{i} / 2$ and at the roots of $1+x+7 x^{2}$ (see [6] for further details), but since these singularities lie in the complex plane outside the physical disc $|x| \leq x_{c}$ their contributions are exponentially suppressed asymptotically. 
We argued in the introduction and demonstrated in Section 4 that $p_{n}$ and $r_{n}$ have exactly the same asymptotic form to leading order. Any differences between the two problems only appear in the sub-dominant correction terms. The dominant correction term for $\mathcal{P}(x)$ is $\propto \log (1-4 x) / \sqrt{1-4 x}$, which is weaker than the first correction term $-F_{3}(x) / 4 \propto(1-4 x)^{-3 / 4}$ for $\mathcal{P}_{\text {Rot }}(x)$. The amplitudes of both these correction terms are negative, namely, $-3 \sqrt{3} /\left(32 \pi^{3 / 2}\right) \approx-0.029$ and $-\frac{1}{4}\left[(1-4 x)^{3 / 4} F_{3}(x)\right]_{x=x_{c}} / \Gamma(3 / 4)=$ $-1 /(16 \sqrt{2} \Gamma(3 / 4)) \approx-0.036$, respectively. So this bears out our intuition that $r_{n} \leq p_{n}$, since the placement of a rotated inner staircase polygon faces more restrictions than the placement of aligned inner and outer staircase polygons. These differences indicate that combinatorial arguments for a proof of sub-dominant behaviour must be quite subtle!

The combinatorial derivation of the expression for $\mathcal{P}_{\text {Rot }}(x)$ turned out to be very interesting. In particular it allowed us to prove exact results for several generalisations of the original model. In the case where we only count the external perimeter we find a change in the value of dominant singularity to $x_{c}=4 / 25$. There is still a singularity at $x=1 / 4$ and interestingly the behaviour at this (now sub-dominant) critical point is much more complicated than at the leading singularity. We also studied the case where the puncture was replaced by a different type of convex polygon (with the external staircase polygon avoiding its minimal bounding rectangle). The major finding of interest was that the dominant singularity remained at $x=x_{c}=1 / 4$. However, the critical exponent changed depending on the asymptotic growth of the generating function of the puncture. When the asymptotic growth of the puncture is slower than or equal to that of staircase polygons we have a simple pole at $x=x_{c}=1 / 4$. However, in the case of directed convex and the case of convex polygons (both of which are asymptotically more numerous than staircase polygons) the critical exponent changes to reflect a faster growth in the number of the associated punctured polygons. Finally, we looked at the case of nested staircase polygons in which case we can derive formulas for a fixed number $k$ of nested staircase polygons. We found that the singularity remains at $x=1 / 4$ with a leading exponent $-2+1 / 2^{k-1}$; while we have observed this for $1 \leq k \leq 10$, we have not proved it.

Another interesting open question is the behaviour of staircase polygons with an arbitrary number of nested polygons (that is the function formed by summing $\mathcal{P}_{k}(x)$ ). This work can also be extended to different types of outer polygons. The method of Section 5 should generalise to other types of convex polygons. Also it might be interesting to look at counts by area as well.

\section{E-mail or WWW retrieval of series}

The series for the generating function studied in this paper can be obtained via e-mail by sending a request to I.Jensen@ms.unimelb.edu.au or via the world wide web on the URL http://www.ms.unimelb.edu.au/ iwan// by following the instructions. 


\section{Acknowledgments}

We would like to thank M Zabrocki for his comments on the combinatorial constructions

in Section 5 and C Richard and A J Guttmann for their useful comments on the manuscript. IJ gratefully acknowledge financial support from the Australian Research Council. AR gratefully acknowledges financial support from NSERC Canada.

\section{References}

[1] Bousquet-Mélou M 1996 A method for the enumeration of various classes of column-convex polygons Disc. Math. 154 1-25

[2] Richard C, Guttmann A J and Jensen I 2001 Scaling function and universal amplitude combinations for self-avoiding polygons J. Phys. A: Math. Gen. 34 L495-L501

[3] Richard C 2002 Scaling behaviour of two-dimensional polygon models J. Stat. Phys. 108 459-493

[4] Richard C, Jensen I and Guttmann A J 2004 Scaling function for self-avoiding polygons revisited J. Stat. Mech.: Th. Exp. P08007

[5] Guttmann A J, Jensen I, Wong L H and Enting I G 2000 Punctured polygons and polyominoes on the square lattice J. Phys. A: Math. Gen. 33 1735-1764

[6] Guttmann A J and Jensen I 2006 The perimeter generating function of punctured staircase polygons J. Phys. A: Math. Gen. 39 3871-3882

[7] Richard C, Jensen I and Guttmann A J 2007 Area distribution and scaling function for punctured polygons Preprint: math.CO/0701633

[8] Enting I G 1980 Generating functions for enumerating self-avoiding rings on the square lattice $J$. Phys. A: Math. Gen. 13 3713-3722

[9] Guttmann A J and Jensen I 2006 Fuchsian differential equation for the perimeter generating function of three-choice polygons Séminaire Lotharingien de Combinatoire 54 B54c Preprint: math.CO/0506317

[10] Zenine N, Boukraa S, Hassani S and Maillard J M 2004 The Fuchsian differential equation of the square lattice Ising model $\chi^{(3)}$ susceptibility J. Phys. A: Math. Gen. 37 9651-9668

[11] Zenine N, Boukraa S, Hassani S and Maillard J M 2005 Square lattice Ising model susceptibility: series expansion method and differential equation for $\chi^{(3)}$ J. Phys. A: Math. Gen. 38 1875-1899

[12] Zenine N, Boukraa S, Hassani S and Maillard J M 2005 Ising model susceptibility: the Fuchsian differential equation for $\chi^{(4)}$ and its factorization properties J. Phys. A: Math. Gen. $384149-$ 4173

[13] Ince E L 1927 Ordinary differential equations (London: Longmans, Green and Co. Ltd.)

[14] Forsyth A R 1902 Part III. Ordinary linear equations vol. IV of Theory of differential equations. (Cambridge: Cambridge University Press) 